Bundesgesundheitsbl - Gesundheitsforsch Gesundheitsschutz 2008 · 51:1219 DOI 10.1007/s00103-008-0682-7

(c) Springer Medizin Verlag 2008

Mitteilungen des Arbeitskreises Blut des Bundesministeriums für Gesundheit

\title{
Meldung von Spendern mit Antikörpern gegen das Hepatitis-B-Core- Antigen (Anti-HBC)
}

\section{Bei der 66. Sitzung des Arbeitskreises Blut am 09. Juni 2008 wurde folgendes Votum (V 37) verabschiedet:}

as Votum 31 des Arbeitskreises Blut empfiehlt eine Untersuchung der Blutund Plasmaspenden auf Anti-HBc zur Erhöhung der Sicherheit von zellulären Blutkomponenten und quarantänegelagertem Frischplasma. Mit Inkrafttreten des Bescheides des Paul-Ehrlich-Instituts (Abwehr von Arzneimittelrisiken; Testung auf Antikörper gegen Hepatitis-B-CoreAntigen im Blutspendewesen) ist die Testung der Spender, aus deren Spende zelluläre Blutkomponenten und quarantänegelagertes Frischplasma gewonnen wird, auf Anti-HBc seit dem 30.9.2006 verbindlich geworden.

Anti-HBc-positive Spenden, die Hepatitis-B-Oberflächen-Antigen (HBsAg) und HBV-DNA-negativ sind, werden derzeit von den infektionsepidemiologischen Meldungen nach $₫ 22$ Transfusionsgesetz (TFG) nicht erfasst. Eine Abschätzung der Gesamtprävalenz von HBV-Infektionen und eine Beurteilung des Zugewinns an Informationen durch Einführung der Testung auf Anti-HBc sind daher bislang nicht möglich.

Der AK Blut empfiehlt daher eine (kumulative) Übermittlung aller Befunde zu Anti-HBc-positiven, HBsAg-negativen Spendern, bzw. zu allen Anti-HBc-positiven Neuspendern, die nicht weiter abge- klärt und von der Spende ausgeschlossen wurden, an das Robert Koch-Institut. Die Meldung sollte zusammen mit den infek-

\begin{tabular}{|l|l}
\hline Geburtsjahr & Anti-HBs-Titer \\
\hline Geschlecht & Ergebnis der Pool-HBV-NAT \\
\hline $\begin{array}{l}\text { Spendertyp } \\
\text { (Erst/Mehrfachspender) }\end{array}$ & Ergebnis der Einzel-HBV-NAT \\
\hline Spendenart & $\begin{array}{l}\text { Sensitivität der verwendeten NAT-Verfahren } \\
\text { bezogen auf die Einzelspende }\end{array}$ \\
\hline $\begin{array}{l}\text { Anti-HBs qualitativ } \\
\text { (d.h. positiv, negativ, nicht durchgeführt) }\end{array}$ & Verwendetes Anti-HBc-Testverfahren \\
\hline
\end{tabular}

Die Daten sollen durch die Träger der Einrichtungen, getrennt nach den einzelnen Spendeeinrichtungen, jährlich in Form einer Liste jeweils zum 31.3. des nachfolgenden Jahres gemeldet werden. Nicht durchgeführte Untersuchungen sollen mit dem Kürzel „n.d.“ angegeben werden. Die Auflistung der Daten sollte in einem gängigen EDV-Format (z.B. Microsoft-Excel) erfolgen. Eine entsprechende Vorlage kann das RKI zur Verfügung stellen. Die Übermittlung der Daten sollte nach Möglichkeit elektronisch erfolgen. Um eine vollständige Erfassung der Daten seit Umsetzung des Votums 31 zu gewährleisten, ist eine rückwirkende Meldung der Daten von 2006 notwendig.

Ist bei einem HBs-Ag negativen, anti- tionsepidemiologischen Meldungen unter Angabe folgender Parameter erfolgen:

HBc-positiven Spender die HBV-NAT positiv, so ist dieser Befund im Rahmen der infektionsepidemiologischen Meldungen nach $\$ 22$ TFG als bestätigt positiver Befund auf dem Quartalsbogen sowie mittels Spenderdatenbogen zu melden.

Die mit der oben genannten Liste erhobenen Daten gehen (mit Ausnahme der bestätigt NAT-positiven Befunde, s.o.) nicht in die jährliche Berechnung der Infektionshäufigkeiten ein. Sie werden separat ausgewertet und dargestellt.

Für den Arbeitskreis Blut:

Prof. Dr. R. Burger, Vorsitzender

Dr. R. Offergeld, Geschäftsführerin 